\title{
Fuzzy-Model-Based Control for Markov Switching Singularly Perturbed Systems with the Stochastic Communication Protocol
}

\author{
Zhiguo An, ${ }^{1,2}$ Qijuan Chen ${ }^{(D,}{ }^{1}$ Junxiang Liu, ${ }^{2}$ Le Luan, ${ }^{2}$ Yong Wang, ${ }^{2}$ Kai Zhou, ${ }^{2}$ \\ Wenxiong Mo, ${ }^{2}$ and Huihong Huang ${ }^{2}$ \\ ${ }^{1}$ School of Power and Mechanical Engineering, Wuhan University, Wuhan, China \\ ${ }^{2}$ Guangzhou Power Supply Bureau, Guangdong Power Grid Co., Ltd., Guangzhou, China \\ Correspondence should be addressed to Qijuan Chen; qjchen@whu.edu.cn
}

Received 16 December 2020; Revised 21 January 2021; Accepted 1 February 2021; Published 18 February 2021

Academic Editor: Shihong Ding

Copyright (c) 2021 Zhiguo An et al. This is an open access article distributed under the Creative Commons Attribution License, which permits unrestricted use, distribution, and reproduction in any medium, provided the original work is properly cited.

This work is concerned with the $H_{\infty}$ control for Markov switching singularly perturbed systems with the stochastic communication protocol. To coordinate the data transmission and save the bandwidth usage, the stochastic communication protocol with a compensator is applied to schedule the information exchange. The goal of this work is to design a joint-Markov-processbased controller such that the resulting system is stochastically stable with prescribed performance. Based on the Lyapunov functional technique, a sufficient condition is derived to ensure the existence of the achieved controller. Finally, the effectiveness and correctness of the developed results are verified by the simulation example.

\section{Introduction}

As a significant component of hybrid systems, Markov switching systems (MSSs) have gained extensive interest due to their capability in modeling subsystems [1-4]. Note that MSSs consist of a finite number of subsystems, and some abrupt variations can be depicted by a Markov process, which is recognized as a key feature of MSSs. Nowadays, owing to their potential practical application, much effort has been devoted, and wonderful fruitful achievements have been gained for both continuous-time MSSs and discrete-time cases [5-8]. Nevertheless, as pointed out in $[5,6]$, the most existing results are concerned with Markov switching linear systems. Due to the widespread of nonlinear characteristics, it is natural to investigate the Markov switching nonlinear systems. Compared with the standard MSSs, the Markov switching nonlinear systems are more general as they contain high nonlinearity. Lately, the T-S fuzzy model has been tendered to deal with the system's nonlinearities $[9,10]$. Benefit from the T-S fuzzy model, many Markov switching nonlinear systems can be approximated as T-S fuzzy MSSs (FMSSs). Following this excellent result, quantities of valuable results have been forwarded on T-S FMSSs [11-13]. For instance, in [11], a dropout compensation approach has been studied for T-S FMSSs. With respect to the network-induced phenomena, the cyber attack has been considered in FMSSs [13].

In many dynamic systems, the system behaviors are involved in multiple-time-scale property. The parasitic parameters, for instance, small-time constants and inductances, may result in the numerical ill-conditioned issues of physical systems. In this regard, the singular perturbation strategy has been employed to tackle the above obstacles. Thanks to singularly perturbed systems (SPSs), the multipletime-scale-based systems can be transformed into a framework model. Note that the examples of SPSs can be widely found in power systems, airplane systems, etc. Recently, many scholars have drawn their attention to both continuous-time SPSs and discrete-time cases [14-16]. When investigating the SPSs, an extra phenomenon can be encountered, for example, the sudden changes of parameters. To tackle this occurrence, Markov switching SPSs have been studied in $[17,18]$. However, the aforementioned results are concerned with linear systems, little attention has been devoted to T-S fuzzy Markov switching SPSs (FMSSPS) except for $[19,20]$, and this issue remains open and a challenge, which deserves further research. 
In the networked control systems (NCSs), massive signals are communicated through a shared wireless network. As an unavoidable phenomenon, the NCSs always experience data collisions, fading channels, and input saturation [21]. To prevent the above shortage and mitigate the side effects, many communication protocols have been addressed to govern which sensors can obtain access to send signals such as the popular communication schedule called roundrobin protocol [22, 23], try-once-discard protocol [24], and stochastic communication protocol (SCP) [25, 26]. Among them, the SCP is known as an effective method to schedule the signal exchange via a shared channel, in which only one sensor is activated to transmit data. Nevertheless, to our knowledge, no one carries out the exploration of FMSSPSs with the SCP mechanism, which motivates us to this work.
Inspired by the aforementioned discussions, our attention focuses on the control issue for FMSSPSs with the communication protocol. The main contributions are outlined as follows: in light of discrete-time FMSSPSs, to coordinate the data transmission and save the bandwidth usage, the SCP is applied to schedule the information exchange. Benefit from the novel Markov process, a mode-dependent Lyapunov functional is formulated such that the resulting system is stochastically stable, and the controller is designed.

\section{Problem Formulations}

Consider the ith discrete-time Markov switching system modeled by the T-S fuzzy model.

Plant Rule $p$ : IF $\xi_{1}(k)$ is $M_{p 1}$, and $\xi_{2}(k)$ is $M_{p 2}$, and $\cdots$, and $\xi_{g}(k)$ is $M_{p g}$, THEN

$$
\left\{\begin{array}{l}
x_{1}(\iota+1)=A_{p, \varphi(l)}^{11} x_{1}(\iota)+\varepsilon A_{p, \varphi(\iota)}^{12} x_{2}(\iota)+B_{p, \varphi(\iota)}^{1} u(\iota)+C_{p, \varphi(\iota)}^{1} \omega(\iota), \\
x_{2}(\iota+1)=A_{p, \varphi(\iota)}^{21} x_{1}(\iota)+\varepsilon A_{p, \varphi(\iota)}^{22} x_{2}(\iota)+B_{p, \varphi(\iota)}^{2} u(\iota)+C_{p, \varphi(\iota)}^{2} \omega(\iota), \\
z(\iota)=D_{p, \varphi(\iota)}^{1} x_{1}(\iota)+\varepsilon D_{p, \varphi(\iota)}^{2} x_{2}(\iota)+M_{p, \varphi(\iota)} u(\iota)+G_{p, \varphi(\iota)} \omega(\iota),
\end{array}\right.
$$

where $x_{1}(l) \in \mathbb{R}^{n_{s}}$ and $x_{2}(\iota) \in \mathbb{R}^{n_{f}}$ are the fast state and the slow state, respectively. $z(k) \in \mathbb{R}^{n_{z}}$ and $u(\iota) \in \mathbb{R}^{n_{u}}$ are the output vector and control input, respectively. $\omega(\iota) \in l_{2}[0, \infty)$ means the disturbance signal. The sequence $\{\varphi(\iota), \iota \geq 0\}$ renders a discrete-time Markov chain (DTMC) subject to a finite set $\mathcal{N}_{s}=\left\{1,2, \ldots, N_{s}\right\}$. Here, $\varphi(\iota)$ describes a homogeneous DTMC with the transition probability matrix of FMSSPS (1) inferred as

$$
\pi_{i j}=\operatorname{Pr}\{\varphi(\iota+1)=j \mid \varphi(\iota)=i\},
$$

where $\quad \pi_{i j} \geq 0, \quad \sum_{j \in \mathcal{N}_{s}} \pi_{i j}=1, \quad \forall i, j \in \mathcal{N}_{s}, \quad$ and TPM $\Pi=\left[\pi_{i j}\right]_{\mathcal{N}_{s} \times \mathcal{N}_{s}}$.
For technique analysis, $\forall i \in \mathcal{N}_{s}, A_{p, \varphi(l)}^{11}, A_{p, \varphi(l)}^{12}, A_{p, \varphi(l)}^{21}$, $A_{p, \varphi(l)}^{22}, B_{p, \varphi(\iota)}^{1}, B_{p, \varphi(\iota)}^{2}, C_{p, \varphi(l)}^{1}, C_{p, \varphi(l)}^{2}, D_{p, \varphi(l)}^{1}, D_{p, \varphi(l)}^{2}, M_{p, \varphi(l)}$, and $G_{p, \varphi(\iota)}$ are denoted by $A_{p i}^{11}, A_{p i}^{12}, A_{p i}^{21}, A_{p i}^{22}, B_{p i}^{1}, B_{p i}^{2}, C_{p i}^{1}$, $C_{p i}^{2}, D_{p i}^{1}, D_{p i}^{2}, M_{p i}$, and $G_{p i}$, respectively.

Recall the fuzzy weighting function $\hbar_{p}(\xi(\iota))=\left(\prod_{s=1}^{t}\right.$ $\left.M_{p s}\left(\xi_{s}(\iota)\right)\right) /\left(\sum_{p=1}^{r} \prod_{s=1}^{t} M_{p s}\left(\xi_{s}(\iota)\right)\right)$, where $M_{p s}\left(\xi_{s}(\iota)\right)$ refers to the grade of the membership degree of $\xi_{s}(\iota)$ in $M_{p s}$. In general, assume $\sum_{p=1}^{r} \hbar_{p}(\xi(\iota))=1$ and $\hbar_{p}(\xi(\iota)) \geq 0$.

Let $x(\iota)=\left[\begin{array}{lll}x_{1}^{\top}(l) & x_{2}^{\top}(\iota)\end{array}\right]^{\top}$; by virtue of T-S fuzzy technique, FMSSPS (1) is derived as

$$
\left\{\begin{array}{l}
x(\iota+1)=\sum_{p=1}^{r} \hbar_{p}(\xi(\iota))\left[A_{p i} E_{\varepsilon} x(\iota)+B_{p i} u(\iota)+C_{p i} \omega(\iota)\right] \\
z(\iota)=\sum_{p=1}^{r} \hbar_{p}(\xi(\iota))\left[D_{p i} E_{\varepsilon} x(\iota)+M_{p i} u(\iota)+G_{p i} \omega(\iota)\right]
\end{array}\right.
$$

where $E_{\epsilon}=\operatorname{diag}\left\{I_{n_{s}}, \epsilon I_{n_{f}}\right\}, A_{p i}=\left[\begin{array}{cc}A_{p i}^{11} & A_{p i}^{12} \\ A_{p i}^{21} & A_{p i}^{22}\end{array}\right], B_{p i}=\left[\begin{array}{c}B_{p i}^{1} \\ B_{p i}^{2}\end{array}\right]$, $C_{p i}=\left[\begin{array}{c}C_{p i}^{1} \\ C_{p i}^{2}\end{array}\right]$, and $D_{p i}=\left[\begin{array}{ll}D_{p i}^{1} & D_{p i}^{2}\end{array}\right]$.

In the NCSs, some redundant signals are communicated in the conventional data transmission manner, which may result in unfavorite phenomena, for instance, data collisions. The control signal $v(k)$ and the actuators $u(k)$ share the same communication network $(\mathrm{CN})$. To prevent such unfavorite factors, the SCP scheduling is used to regulate the node order in transmitting data. Note that only one sensor is borrowed to release the signal each time, and the sensors are chosen in a stochastic way. In general, letting $\psi(\iota) \in\{1,2$, $\left.\ldots, N_{c}\right\}$ signifies the chosen actuator which gains the permission to access the $\mathrm{CN}$ at the time interval $\iota$. Notably, $\psi(\iota)$ can be recognized as a stochastic process regulated by another DTMC obeying a set $\mathcal{N}_{c}=\left\{1,2, \ldots, N_{c}\right\}$, and TPM $\Psi=\left[\tau_{m n}\right]_{N_{c} \times N_{c}}$ is determined by

$$
\tau_{m n}=\operatorname{Pr}\{\psi(\iota+1)=n \mid \psi(\iota)=m\},
$$

where $\forall m, n \in \mathcal{N}_{c}, \tau_{m n} \in[0,1]$, and $\sum_{n \in \mathcal{N}_{c}} \tau_{m n}=1$. 
Let $\quad v(\iota)=\left[\begin{array}{llll}v_{1}^{\top}(\iota) & v_{2}^{\top}(\iota) & \cdots & v_{n_{u}}^{\top}(\iota)\end{array}\right] \quad$ and $u(\iota)=\left[\begin{array}{lllll}u_{1}^{\top}(\iota) & u_{2}^{\top}(\iota) & \cdots & u_{n_{u}}^{\top}(\iota)\end{array}\right]$, where $v_{m}(\iota)$ denotes the $m$ th control input vector and $u_{n}$ signifies the $n$th actuator. Firstly, assume that a set of zero-order hold is employed in the signal transmission. Accordingly, the $m$ th actuator $u_{m}(\iota)$ is updated by the following principle:

$$
u_{m}(\iota)= \begin{cases}v_{m}(\iota), & \text { if } \psi(\iota)=m, \\ u_{m}(\iota-1), & \text { otherwise. }\end{cases}
$$

Aiming at describing the data transmission strategy of actuators mathematically, a Kronecker sign function is inferred as

$$
\delta(x-y)= \begin{cases}1, & \text { if } x=y, \\ 0, & \text { otherwise. }\end{cases}
$$

As indicated from the updating principle (5), the $m$ th actuator $u_{m}(\iota)$ is updated when $\psi(\iota)=m$. Consequently, for $\forall \iota$, the updated actuator $u(\iota)$ can be devised as

$$
u(\iota)=\Psi_{\psi(\iota)} v(\iota)+\left(I-\Psi_{\psi(\iota)}\right) u(\iota-1),
$$

where $\Psi_{m} \triangleq \operatorname{diag}\left\{\delta_{m}^{1}, \delta_{m}^{2}, \ldots, \delta_{m}^{n_{u}}\right\}\left(m=1,2, \ldots, n_{u}\right) \quad$ and $\delta_{x}^{y}=\delta(x-y)$.

The control law $v(k)$ in this work is constructed as follows:

$$
v(\iota)=\sum_{q=1}^{r} \hbar_{q}(\xi(\iota)) K_{q, \varphi(l)} E_{\varepsilon} x(\iota)
$$

where $K_{q, \varphi(l)}$ are matrices to be designed.

Substituting (8) into (3), the closed-loop FMSPS (9) is formulated as

$$
\left\{\begin{array}{l}
x(\iota+1)=\sum_{p=1}^{r} \hbar_{p}(\xi(\iota)) \sum_{q=1}^{r} \hbar_{q}(\xi(\iota))\left[\mathscr{A}_{p q i m} E_{\varepsilon} x(\iota)+B_{p i}\left(I-\Psi_{m}\right) u(\iota-1)+C_{p i} \omega(\iota)\right] \\
z(\iota)=\sum_{p=1}^{r} \hbar_{p}(\xi(\iota)) \sum_{q=1}^{r} \hbar_{q}(\xi(\iota))\left[\mathscr{D}_{p q i m} E_{\varepsilon} x(\iota)+M_{p i}\left(I-\Psi_{m}\right) u(\iota-1)+G_{p i} \omega(\iota)\right]
\end{array}\right.
$$

where

$$
\begin{gathered}
\mathscr{A}_{p q i m}=A_{p i}+B_{p i} \Psi_{m} K_{q i}, \\
\mathscr{D}_{p q i m}=D_{p i}+M_{p i} \Psi_{m} K_{q i} .
\end{gathered}
$$

Before proceeding further, some lemmas and definitions are provided.

Definition 1 (see [27]). The FMSSPS (9) with $\omega(k)=0$ is named stochastic stable (SS) if for any $\left(\delta_{0}, \vartheta_{0}\right)$, one has

$$
\mathrm{E}\left\{\sum_{k=0}^{\infty}\|\delta(k)\|^{2} \mid \delta_{0}, \vartheta_{0}\right\}<\infty .
$$

Definition 2 (see [27]). The FMSSPS (9) is named SS with a prescribed $\mathscr{H}_{\infty}$ performance level $\gamma$ if the FMSSPS (18) is SS and under zero initial condition such that

$$
\sum_{k=0}^{\infty} \mathrm{E}\left\{\|\delta(k)\|^{2}\right\}<\gamma^{2} \sum_{k=0}^{\infty} \mathrm{E}\left\{\|\varrho(k)\|^{2}\right\} .
$$

Lemma 1 (see [18]). For given a scalar $\bar{\epsilon}>0, \mathscr{W}_{1}, \mathscr{W}_{2}$, and $\mathscr{W}_{3}$ are matrices with suitable dimensions. For any $\epsilon \in(0, \bar{\epsilon}]$, $\mathscr{W}_{1}+\epsilon \mathscr{W}_{1}+\epsilon^{2} \mathscr{W}_{1}>0$ such that

$$
\begin{aligned}
& \mathscr{W}_{1}>0, \\
& \mathscr{W}_{1}+\bar{\epsilon} \mathscr{W}_{1}>0, \\
& \mathscr{W}_{1}+\bar{\epsilon} \mathscr{W}_{2}+\bar{\epsilon}^{2} \mathscr{W}_{3}>0 .
\end{aligned}
$$

Lemma 2 (see [18]). For any symmetric matrices $\mathscr{R}_{t}(t=$ $1,2,3,4)$ and matrix $\mathscr{R}_{5}$ which meets

$$
\begin{array}{r}
\mathscr{R}_{1}>0, \\
{\left[\begin{array}{cc}
\mathscr{R}_{1}+\bar{\epsilon} \mathscr{R}_{3} & \bar{\epsilon} \mathscr{R}_{5}^{\top} \\
* & \bar{\epsilon} \mathscr{R}_{2}
\end{array}\right]>0,} \\
{\left[\begin{array}{cc}
\mathscr{R}_{1}+\bar{\epsilon} \mathscr{R}_{3} & \bar{\epsilon} \mathscr{R}_{5}^{\top} \\
* & \bar{\epsilon} \mathscr{R}_{2}+\bar{\epsilon}^{2} \mathscr{R}_{4}
\end{array}\right]>0,}
\end{array}
$$

one has $E_{\epsilon} \mathscr{R}_{\epsilon}=\mathscr{R}_{\epsilon}^{\top} E_{\epsilon}>0$ for any $\epsilon \in(0, \bar{\epsilon}]$, where

$$
\mathscr{R}_{\varepsilon}=\left[\begin{array}{cc}
\mathscr{R}_{1}+\epsilon \mathscr{R}_{3} & \epsilon \mathscr{R}_{5}^{\top} \\
* & \epsilon \mathscr{R}_{2}+\epsilon^{2} \mathscr{R}_{4}
\end{array}\right] .
$$

\section{Main Results}

In this section, sufficient conditions are elicited to ensure the SS and a prescribed $\mathscr{H}_{\infty}$ performance level of the FMSSPS (9).

Theorem 1. The closed FMSSPS (9) is called SS with a prescribed $\mathscr{H}_{\infty}$ performance level $\gamma$ if there exist symmetric matrices $\bar{P}_{i}>0, \bar{Q}_{i}>0, \mathscr{R}_{1}, \mathscr{R}_{2}, \mathscr{R}_{3}$, and $\mathscr{R}_{4}$ and matrices $\mathscr{R}_{5}, \bar{K}_{\epsilon q i}, \mathscr{U}_{1}$, and $\mathscr{U}_{2}$ such that

$$
\begin{aligned}
& \mathscr{R}_{1}>0, \\
& {\left[\begin{array}{cc}
\mathscr{R}_{1}+\bar{\epsilon} \mathscr{R}_{3} & \bar{\epsilon} \mathscr{R}_{5}^{\top} \\
\bar{\epsilon} \mathscr{R}_{5} & \bar{\epsilon} \mathscr{R}_{2}
\end{array}\right]>0, }
\end{aligned}
$$




$$
\begin{gathered}
{\left[\begin{array}{cc}
\mathscr{R}_{1}+\bar{\epsilon} \mathscr{R}_{3} & \bar{\epsilon} \mathscr{R}_{5}^{\top} \\
\bar{\epsilon} \mathscr{R}_{5} & \bar{\epsilon} \mathscr{R}_{2}+\bar{\epsilon}^{2} \mathscr{R}_{4}
\end{array}\right]>0,} \\
\Gamma_{p \text { pim }}(t)<0, \quad(1 \leq p \leq r, t=\ell, 1, \mathscr{J}), \\
\Gamma_{p q i m}(t)+\Gamma_{q p i m}(t)<0, \quad(1 \leq p<q \leq r, t=\ell, 1, \mathscr{J}) .
\end{gathered}
$$

$$
\begin{aligned}
& \Gamma_{p q i m}(t)=\left[\begin{array}{cccc}
\Gamma_{p q i}^{1(t)} & \Gamma_{p q i m}^{2(t) \top} \mathscr{T}_{f} & \Gamma_{p q i m}^{3(t) \top} \mathscr{T}_{f} & \Gamma_{p q i m}^{4(t) \top} \\
* & \Gamma_{p q i}^{5} & 0 & 0 \\
* & * & \Gamma_{p q i}^{6} & 0 \\
* & * & * & -I
\end{array}\right], \quad(t=\ell, 1, \mathscr{J}), \\
& \Gamma_{p q i}^{1(\ell)}=\operatorname{diag}\left\{\bar{P}_{i}-\left(\mathscr{V}_{1}+\mathscr{V}_{1}^{\top}\right), \bar{Q}_{i}-\left(Y_{i}+Y_{i}^{\top}\right),-\gamma^{2} I\right\}, \Gamma_{p q i}^{1(1)}=\operatorname{diag}\left\{\bar{P}_{i}-\left(\mathscr{R}_{\bar{\epsilon}}+\mathscr{R}_{\overline{\bar{\epsilon}}}^{\top}\right), \overline{\mathrm{Qm}}_{i}-\left(Y_{i}+Y_{i}^{\top}\right),-\gamma^{2} I\right\},
\end{aligned}
$$

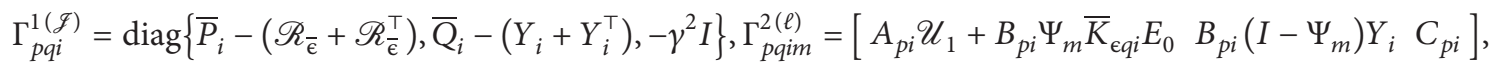

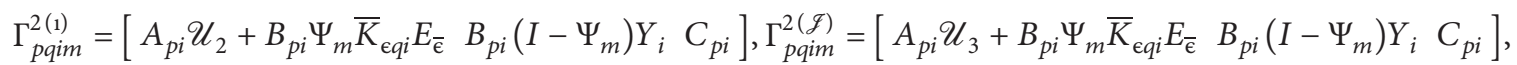

$$
\begin{aligned}
& \Gamma_{p q i m}^{3(\ell)}=\left[\begin{array}{lll}
\Psi_{m} \bar{K}_{e q i} E_{0} & \left(I-\Psi_{m}\right) Y_{i} & 0
\end{array}\right], \Gamma_{p q i m}^{3(1)}=\Gamma_{p q i}^{3(\mathcal{F})}=\left[\begin{array}{lll}
\Psi_{m} \bar{K}_{e q i} E_{\bar{\epsilon}} & \left(I-\Psi_{m}\right) Y_{i} & 0
\end{array}\right], \\
& \Gamma_{p q i m}^{4(\ell)}=\left[\begin{array}{lll}
D_{p i} \mathcal{U}_{1}+M_{p i} \Psi_{m} \bar{K}_{e q i} E_{0} M_{p i}\left(I-\Psi_{m}\right) Y_{i} G_{p i}
\end{array}\right], \Gamma_{p q i m}^{4(1)}=\left[\begin{array}{ll}
D_{p i} \mathcal{U}_{2}+M_{p i} \Psi_{m} \bar{K}_{e q i} E_{\bar{\varepsilon}} & M_{p i}\left(I-\Psi_{m}\right) Y_{i} G_{p i}
\end{array}\right] \text {, } \\
& \Gamma_{p q i m}^{4(\mathcal{F})}=\left[D_{p i} \mathscr{U}_{3}+M_{p i} \Psi_{m} \bar{K}_{e q i} E_{\bar{\epsilon}} M_{p i}\left(I-\Psi_{m}\right) \mathscr{R}_{\bar{\epsilon}} G_{p i}\right], \Gamma_{p q i}^{5}=\operatorname{diag}\left\{-\bar{P}_{1},-\bar{P}_{2}, \ldots,-\bar{P}_{N_{r}}\right\}, \\
& \Gamma_{p q i}^{6}=\operatorname{diag}\left\{-\bar{Q}_{1},-\bar{Q}_{2}, \ldots,-\bar{Q}_{N_{r}}\right\}, \mathscr{T}_{i}=\left[\sqrt{\theta_{f 1}} I \sqrt{\theta_{f 2}} I \cdots \sqrt{\theta_{f N_{r}}} I\right], \mathscr{R}_{\bar{\epsilon}}=\mathscr{V}_{1}+\bar{\epsilon} \mathscr{V}_{2}, \mathscr{U}_{1}=\mathscr{W}_{1}, \\
& \mathscr{U}_{2}=\mathscr{W}_{1}+\bar{\epsilon} \mathscr{W}_{2}, \mathscr{U}_{3}=\mathscr{W}_{1}+\bar{\epsilon} \mathscr{W}_{2}+\bar{\epsilon}^{2} \mathscr{W}_{3}, \mathscr{V}_{1}=\left[\begin{array}{cc}
\mathscr{R}_{1} & 0 \\
\mathscr{R}_{5} & \mathscr{R}_{2}
\end{array}\right], \mathscr{V}_{2}=\left[\begin{array}{cc}
\mathscr{R}_{3} & \mathscr{R}_{5}^{\top} \\
0 & \mathscr{R}_{4}
\end{array}\right], \\
& \mathscr{W}_{1}=\left[\begin{array}{cc}
\mathscr{R}_{1} & 0 \\
0 & 0
\end{array}\right], \mathscr{W}_{2}=\left[\begin{array}{cc}
\mathscr{R}_{3} & \mathscr{R}_{5}^{\top} \\
\mathscr{R}_{5} & \mathscr{R}_{2}
\end{array}\right], \mathscr{W}_{3}=\left[\begin{array}{cc}
0 & 0 \\
0 & \mathscr{R}_{4}
\end{array}\right], E_{0}=\left[\begin{array}{cc}
I_{n_{s}} & 0 \\
0 & 0
\end{array}\right], E_{\bar{\epsilon}}=\left[\begin{array}{cc}
I_{n_{s}} & 0 \\
0 & \bar{\epsilon} I_{n_{i}}
\end{array}\right] \text {. }
\end{aligned}
$$

Proof. Combining with the linear matrix inequalities (LMIs) (17)-(19) and Lemma 2, for any $\epsilon \in(0, \bar{\epsilon}]$, it yields that

$$
\left[\begin{array}{cccc}
\widetilde{\Gamma}_{p q i}^{1} & \widetilde{\Gamma}_{p q i m}^{2 \top} \mathscr{T}_{i} & \widetilde{\Gamma}_{p q i m}^{3 \top} \mathscr{T}_{i} & \widetilde{\Gamma}_{p q i m}^{4 \mathrm{~T}} \\
* & \Gamma_{p q i}^{5} & 0 & 0 \\
* & * & \Gamma_{p q i}^{6} & 0 \\
* & * & * & -I
\end{array}\right]<0,
$$

where

$$
\begin{aligned}
\widetilde{\Gamma}_{p q i}^{1} & =\operatorname{diag}\left\{\bar{P}_{i}-\left(\mathscr{R}_{\epsilon}+\mathscr{R}_{\epsilon}^{\top}\right), \bar{Q}_{i}-\left(\mathscr{R}_{\epsilon}+\mathscr{R}_{\epsilon}^{\top}\right),-\gamma^{2} I\right\}, \\
\widetilde{\Gamma}_{p q i m}^{2} & =\left[\begin{array}{lll}
A_{p i} E_{\epsilon} \mathscr{R}_{\epsilon}+B_{p i} \Psi_{m} K_{q i} \mathscr{R}_{\epsilon}^{\top} E_{\epsilon} & B_{p i}\left(I-\Psi_{m}\right) \mathscr{R}_{\epsilon} & C_{p i}
\end{array}\right], \\
\widetilde{\Gamma}_{p q i m}^{3} & =\left[\begin{array}{lll}
\Psi_{m} K_{q i} \mathscr{R}_{\epsilon}^{\top} E_{\epsilon} & \left(I-\Psi_{m}\right) \mathscr{R}_{\epsilon} & 0
\end{array}\right], \\
\widetilde{\Gamma}_{p q i m}^{4} & =\left[\begin{array}{lll}
D_{p i} E_{\epsilon} \mathscr{R}_{\epsilon}+M_{p i} \Psi_{m} K_{q i} \mathscr{R}_{\epsilon}^{\top} E_{\epsilon} & M_{p i}\left(I-\Psi_{m}\right) \mathscr{R}_{\epsilon} & G_{p i}
\end{array}\right] .
\end{aligned}
$$

Recalling Lemma 2 and LMIs (17)-(19), for any $\epsilon \in(0, \bar{\epsilon}]$, it is clear that $E_{\epsilon} \mathscr{R}_{\epsilon}=\mathscr{R}_{\epsilon}^{\top} E_{\epsilon}>0$. On the contrary, with respect to the fact that inequality $\left(\mathscr{R}_{\epsilon}^{\top}-\bar{P}_{i}\right) P_{i}\left(\mathscr{R}_{\epsilon}-\bar{P}_{i}\right) \geq 0, \quad\left(\mathscr{R}_{\epsilon}^{\top}-\bar{Q}_{i}\right) Q_{i}\left(\mathscr{R}_{\epsilon}-\bar{Q}_{i}\right) \geq 0$, $\bar{P}_{i}=P_{i}^{-1}$, and $\bar{Q}_{i}=Q_{i}^{-1}$, which yields 


$$
\left[\begin{array}{cccc}
\widehat{\Gamma}_{p q i}^{1} & \widehat{\Gamma}_{p q i m}^{2 \top} \mathscr{T}_{i} & \widehat{\Gamma}_{p q i m}^{3 \top} \mathscr{T}_{i} & \widehat{\Gamma}_{p q i m}^{4 \top} \\
* & \Gamma_{p q i}^{5} & 0 & 0 \\
* & * & \Gamma_{p q i}^{6} & 0 \\
* & * & * & -I
\end{array}\right]<0
$$

In the following, a Lyapunov functional for FMSSPS (9) is established:

$$
\begin{aligned}
V(\iota, x(\iota), u(\iota), \varphi(\iota))= & x^{\top}(\iota) P(\varphi(\iota)) x(\iota) \\
& +u^{\top}(k-1) Q(\varphi(\iota)) u(k-1) .
\end{aligned}
$$

where

$$
\begin{aligned}
& \widehat{\Gamma}_{p q i}^{1}=\operatorname{diag}\left\{-\mathscr{R}_{\epsilon}^{\top} P_{i} \mathscr{R}_{\epsilon},-\mathscr{R}_{\epsilon}^{\top} Q_{i} \mathscr{R}_{\epsilon},-\gamma^{2} I\right\}, \\
& \widehat{\Gamma}_{p q i m}^{2}=\left[\begin{array}{llll}
\mathscr{A}_{p q i} E_{\epsilon} \mathscr{R}_{\epsilon} & B_{p i}\left(I-\Psi_{m}\right) \mathscr{R}_{\epsilon} & C_{p i}
\end{array}\right], \\
& \widehat{\Gamma}_{p q i m}^{3}=\left[\begin{array}{lll}
\Psi_{m} K_{q i} E_{\epsilon} \mathscr{R}_{\epsilon} & \left(I-\Psi_{m}\right) \mathscr{R}_{\epsilon} & 0
\end{array}\right], \\
& \widehat{\Gamma}_{p q i m}^{4}=\left[\begin{array}{lll}
\mathscr{D}_{p q i} E_{\epsilon} \mathscr{R}_{\epsilon} & M_{p i}\left(I-\Psi_{m}\right) \mathscr{R}_{\epsilon} & G_{p i}
\end{array}\right], \\
& \widehat{\Gamma}_{p q i}^{5}=\operatorname{diag}\left\{-P_{1}^{-1},-P_{2}^{-1}, \ldots,-P_{N_{s}}^{-1}\right\}, \\
& \widehat{\Gamma}_{p q i}^{6}=\operatorname{diag}\left\{-Q_{1}^{-1},-Q_{2}^{-1}, \ldots,-Q_{N_{s}}^{-1}\right\} \text {. }
\end{aligned}
$$

Premultiplying and postmultiplying (25) with $\operatorname{diag}\left\{\mathscr{R}_{\epsilon}^{-\top}, \mathscr{R}_{\epsilon}^{-\top}, I, \ldots, I\right\}$ and its transpose, where $\mathscr{R}_{\epsilon}=\mathscr{U}_{1}+\epsilon \mathscr{U}_{2}$, yield

$$
\left[\begin{array}{cccc}
\bar{\Gamma}_{p q i}^{1} & \bar{\Gamma}_{p q i m}^{2 \top} \mathscr{T}_{i} & \bar{\Gamma}_{p q i m}^{3 \top} \mathscr{T}_{i} & \bar{\Gamma}_{p q i m}^{4 \top} \\
* & \Gamma_{p q i}^{5} & 0 & 0 \\
* & * & \Gamma_{p q i}^{6} & 0 \\
* & * & * & -I
\end{array}\right]<0,
$$
has

By calculating the difference of $V(\iota, x(\iota), u(\iota), \varphi(\iota))$, one

$$
\begin{aligned}
\mathscr{E}\{\Delta V(\iota)\}= & \mathscr{E}\{V(\iota+1, x(\iota+1), u(\iota+1), \\
\varphi(\iota+1)= & g \mid \iota, x(\iota), u(\iota), f)\} \\
& -V(\iota, x(\iota), u(\iota), \varphi(\iota)) .
\end{aligned}
$$

Recalling FMSSPS (9), $\mathscr{E}\{\Delta V(\iota)\}$ can be derived as

$$
\begin{aligned}
\mathscr{E}\{\Delta V(\iota)\}= & x^{\top}(\iota+1) \mathscr{P}_{i} x(\iota+1) \\
& -x^{\top}(\iota) P_{i} x(\iota)+u^{\top}(\iota) \mathcal{Q}_{i} u(\iota) \\
& -u^{\top}(k-1) Q_{i} u(k-1),
\end{aligned}
$$

where

$$
\begin{aligned}
\mathscr{P}_{i} & =\sum_{j \in \mathcal{N}_{s}} \pi_{i j} P_{j}, \\
Q_{i} & =\sum_{j \in \mathcal{N}_{s}} \theta_{i j} Q_{j} .
\end{aligned}
$$

In (31), the first term can be further devised as

$$
\begin{aligned}
x^{\top}(\iota+1) \mathscr{P}_{i} x(\iota+1) & \sum_{p=1}^{r} \hbar_{p}(\xi(\iota)) \sum_{q=1}^{r} \hbar_{q}(\xi(\iota)) \\
& {\left[\begin{array}{c}
x^{\top}(\iota)\left(P_{i}+E_{\epsilon} \mathscr{A}_{p q i m}^{\top} \mathscr{P}_{i} \mathscr{A}_{p q i m} E_{\epsilon}\right) x(\iota) \\
+x^{\top}(\iota) E_{\epsilon} \mathscr{A}_{p q i m}^{\top} \mathscr{P}_{i} B_{p i}\left(I-\Psi_{m}\right) u(\iota-1)+u^{\top}(\iota-1)\left(I-\Psi_{m}\right) B_{p i}^{\top} \mathscr{P}_{i} \mathscr{A}_{p q i m} E_{\epsilon} x(\iota) \\
+x^{\top}(\iota) E_{\epsilon} \mathscr{A}_{p q i m}^{\top} \mathscr{P}_{i} C_{p i} \omega(k)+\omega^{\top}(k) C_{p i}^{\top} \mathscr{P}_{i} \mathscr{A}_{p q i m} E_{\epsilon} x(\iota) \\
+u^{\top}(\iota-1)\left(I-\Psi_{m}\right)^{\top} B_{p i}^{\top} \mathscr{P}_{i} B_{p i}\left(I-\Psi_{m}\right) u(\iota-1) \\
+u^{\top}(\iota-1)\left(I-\Psi_{m}\right) B_{p i}^{\top} \mathscr{P}_{i} C_{p i} \omega(k) \\
+\omega^{\top}(k) C_{p i}^{\top} \mathscr{P}_{i} B_{p i}\left(I-\Psi_{m}\right) u(\iota-1)+\omega^{\top}(k) C_{p i}^{\top} \mathscr{P}_{i} C_{p i} \omega(k)
\end{array}\right] . }
\end{aligned}
$$


Besides, the third term in (31) can be rewritten as

$$
\begin{aligned}
u^{\top}(\iota) \mathbb{Q}_{i} u(\iota)= & \sum_{q=1}^{r} \hbar_{q}(\xi(\iota))\left[\Psi_{m} K_{q i} E_{\epsilon} x(\iota)+\left(I-\Psi_{m}\right) u(\iota-1)\right]^{\top} \mathscr{Q}_{i} \\
& \times\left[\Psi_{m} K_{q i} E_{\epsilon} x(\iota)+\left(I-\Psi_{m}\right) u(\iota-1)\right] \\
= & \sum_{q=1}^{r} \hbar_{q}(\xi(\iota))\left[\begin{array}{c}
x^{\top}(\iota) E_{\epsilon} K_{q i}^{\top} \Psi_{m}^{\top} Q_{i} \Psi_{m} K_{q i} E_{\epsilon} x(\iota) \\
+x^{\top}(\iota) E_{\epsilon} K_{q i}^{\top} \Psi_{m}^{\top} Q_{i}\left(I-\Psi_{m}\right) u(\iota-1) \\
+u^{\top}(\iota-1)\left(I-\Psi_{m}\right) Q_{i} \Psi_{m} K_{q i} E_{\epsilon} x(\iota) \\
+u^{\top}(\iota-1)\left(I-\Psi_{m}\right) Q_{i}\left(I-\Psi_{m}\right) u(\iota-1)
\end{array}\right] .
\end{aligned}
$$

Combining (29)-(34) yields

$\mathscr{E}\{\Delta V(\iota)\}=\mathcal{\vartheta}^{\top}(k) \sum_{p=1}^{r} \hbar_{p}(\xi(\iota)) \sum_{q=1}^{r} \hbar_{q}(\xi(\iota)) \vec{\Gamma}_{p q i m} \vartheta(k)$,

where

$$
\begin{aligned}
& \vartheta(k)=\left[\begin{array}{lll}
x^{\top}(\iota) & u^{\top}(\iota-1) & \omega^{\top}(k)
\end{array}\right], \\
& \vec{\Gamma}_{p q i}^{1}=\operatorname{diag}\left\{-P_{i},-Q_{i}, 0\right\} \text {, } \\
& \vec{\Gamma}_{\text {pqim }}=\vec{\Gamma}_{p q i}^{1}+\bar{\Gamma}_{\text {pqim }}^{2 \top} \mathscr{P}_{i} \bar{\Gamma}_{\text {pqim }}^{2}+\bar{\Gamma}_{\text {pqim }}^{3 \top} Q_{i} \bar{\Gamma}_{\text {pqim }}^{3} .
\end{aligned}
$$

When $\omega(k)=0$, it follows from inequality (35) that

$$
\begin{aligned}
\mathscr{E}\{\Delta V(\iota)\} & \leq \overleftarrow{\vartheta}^{\top}(\iota) \sum_{p=1}^{r} \hbar_{p}(\xi(\iota)) \sum_{q=1}^{r} \hbar_{q}(\xi(\iota)) \overleftarrow{\Gamma}_{p q i m} \overleftarrow{\vartheta}(\iota) \\
& \leq-\chi \mathscr{E}\left\{\|x(\iota)\|^{2}\right\},
\end{aligned}
$$

where $\overleftarrow{\vartheta}(k)=\left[x^{\top}(\iota) u^{\top}(l-1)\right], \quad \overleftarrow{\Gamma}_{p q i m}=\overleftarrow{\Gamma}_{p q i}^{1}+\overleftarrow{\Gamma}_{p q i m}^{2 \top} \mathscr{P}_{i}$ $\stackrel{\leftarrow}{\Gamma}_{\text {pqim }}^{2}+\overleftrightarrow{\Gamma}_{\text {pqim }}^{3 \top} Q_{i} \stackrel{\leftrightarrow}{\Gamma}_{\text {pqim }}^{3}, \stackrel{\leftarrow}{\Gamma}_{p q i}^{1}=\operatorname{diag}\left\{-P_{i},-Q_{i}\right\}, \overleftarrow{\Gamma}_{\text {pqim }}^{2}=\left[\mathscr{A}_{\text {pqim }}\right.$ $\left.E B_{p i}\left(I-\Psi_{m}\right)\right], \quad \stackrel{\leftarrow}{\Gamma}_{p q i m}^{3}=\left[\mathscr{D}_{p q i m} E_{\epsilon} M_{p i}\left(I-\Psi_{m}\right)\right]$, and $\chi=$ $\min _{f \in \mathcal{N}_{s}, p, q \in\{1,2, \ldots, r\}}\left\{\lambda_{\text {min }}\left(\overleftarrow{\Gamma}_{p q i m}\right)\right\}$. Clearly, recalling (27), one gets $\chi>0$. Consequently, one concludes that

$$
\begin{aligned}
\mathscr{E}\left\{\sum_{l=0}^{\infty}\|x(\iota)\|^{2}\right\} & <-\frac{1}{\chi} \mathscr{E}\left\{\sum_{l=0}^{\infty} \Delta V(\iota)\right\} \\
& \leq \frac{1}{\chi} \mathscr{E}\{V(0, x(0), u(0), r(0))\}<\infty .
\end{aligned}
$$
SS.

Recalling Definition 1, when $\omega(k)=0$, FMSSPS (16) is

Next, in the case of $\omega(k) \neq 0$, we will provide the analysis of $H_{\infty}$ performance for FMSSPS (16). Define the $H_{\infty}$ performance index:

$$
\mathscr{J}(T)=\mathscr{E}\left\{\sum_{k=0}^{T} z^{\top}(\iota) z(\iota)-\gamma^{2} \omega^{\top}(\iota) \omega(\iota)\right\}
$$

Substituting (35) into (39), $\mathscr{J}(T)$ can be formulated as

$$
\begin{aligned}
\mathscr{J}(T) & \leq \mathscr{E}\left\{\sum_{k=0}^{T}\left[z^{\top}(\iota) z(\iota)-\gamma^{2} \omega^{\top}(\iota) \omega(\iota)+\Delta V(\iota)\right]\right\} \\
& \leq \vartheta^{\top}(\iota) \sum_{p=1}^{r} \hbar_{p}(\xi(\iota)) \sum_{q=1}^{r} \hbar_{q}(\xi(\iota)) \Gamma_{p q i m}^{\prime} \vartheta(\iota),
\end{aligned}
$$

where 

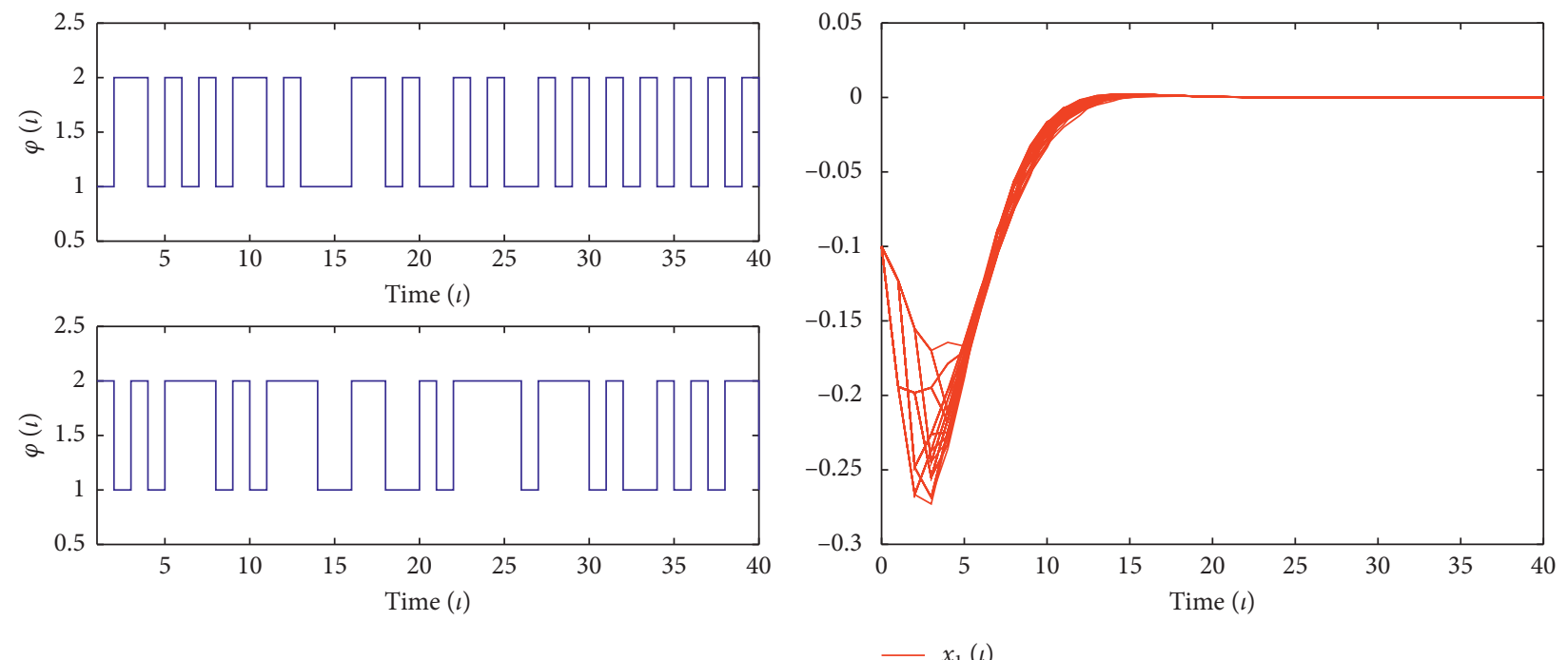

(a)

(b)
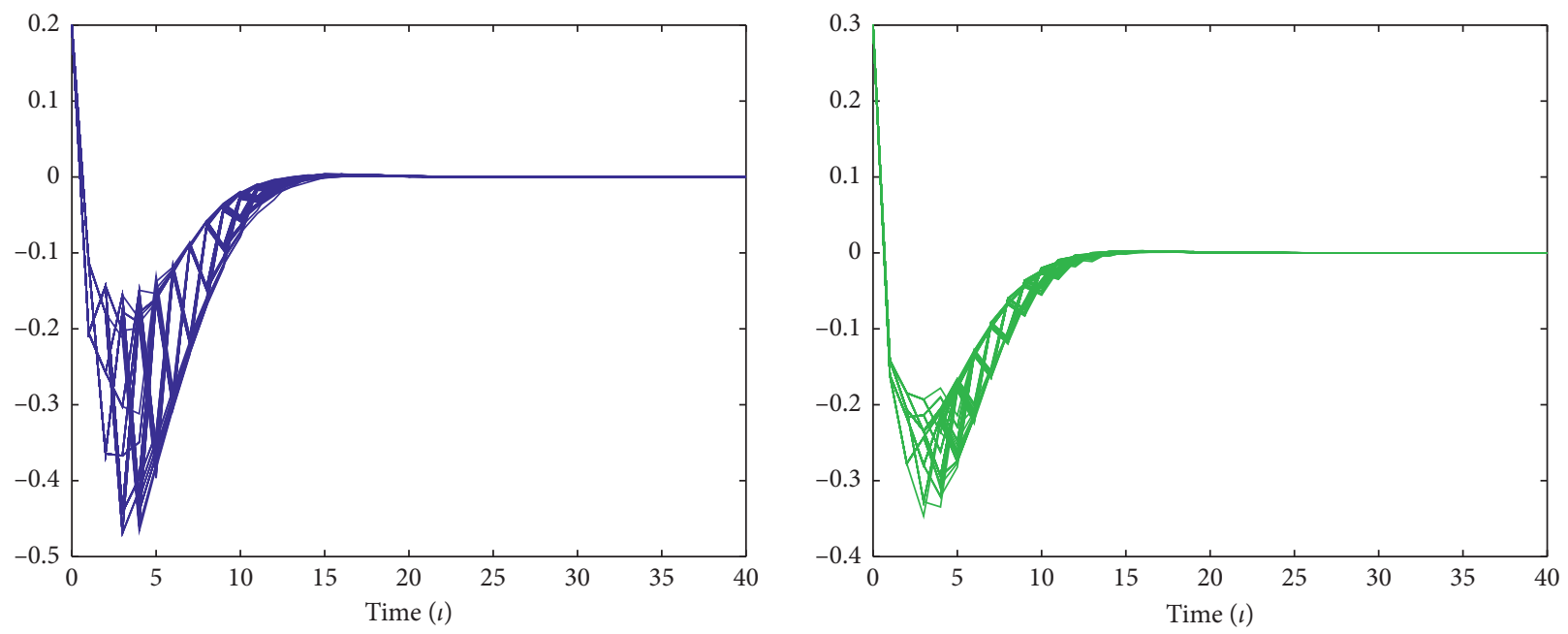

$-x_{2}(\iota)$

$-x_{3}(\iota)$

(c)

(d)

FIgUre 1: The dynamics of FMSSPS (18) in Example 1. (a) The possible mode switching of $\mathrm{MP}^{\prime} \varphi(\iota)$. (b) The evolution of state $x_{1}(\iota)$. (c) The evolution of state $x_{2}(l)$. (d) The evolution of state $x_{3}(l)$.

$$
\begin{aligned}
\Gamma_{\text {pqim }}^{\prime} & =\vec{\Gamma}_{\text {pqim }}+\Gamma_{\text {pqim }}^{4 \top} \Gamma_{\text {pqim }}^{4}, \Gamma_{\text {pqim }}^{4} \\
& =\left[\begin{array}{lll}
\mathscr{D}_{\text {pqim }} E_{\epsilon} & M_{p i}\left(I-\Psi_{m}\right) G_{p i}
\end{array}\right] .
\end{aligned}
$$

Additionally, by applying the Schur complement to (27) and (40), one gets

$$
\mathscr{J}(T)<0 .
$$




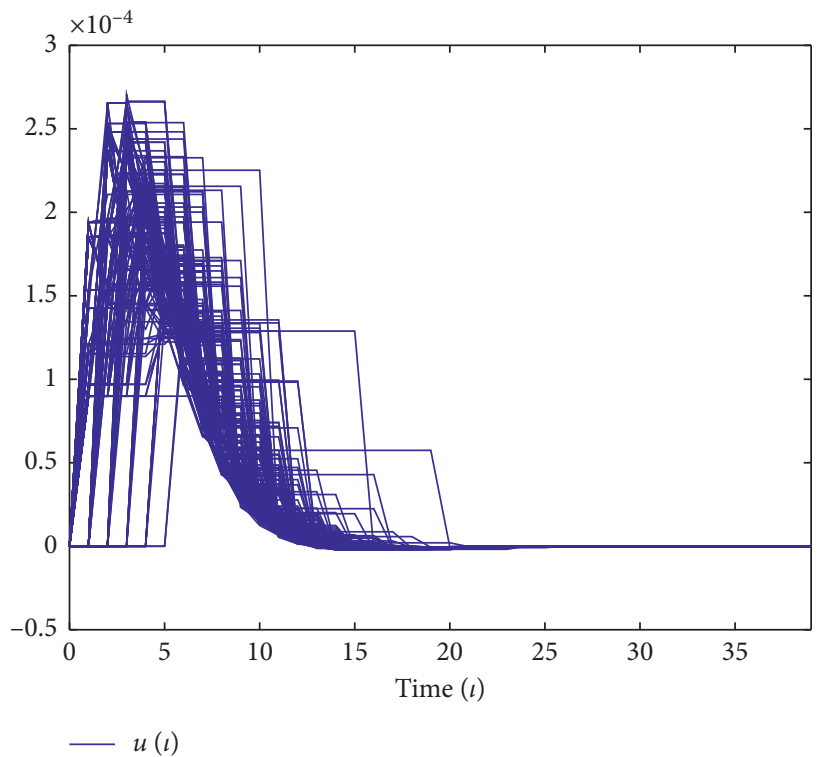

Figure 2: The control input $u(\iota)$ over 100 realizations in Example 1.

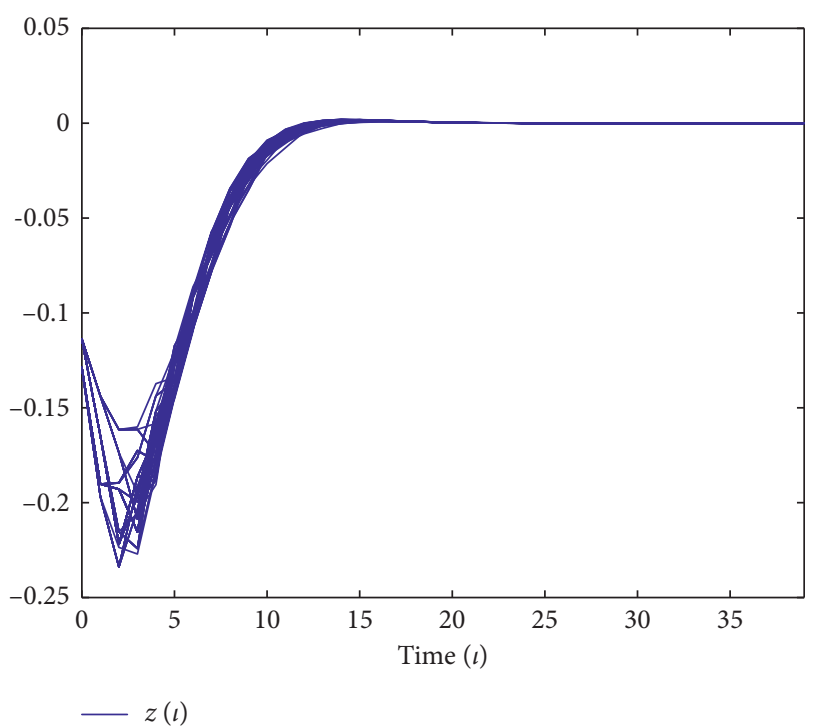

Figure 3: The output $z(\iota)$ over 100 realizations in Example 1.

Letting $T \longrightarrow \infty$, it is directly attained from (42) that

$$
\sum_{l=0}^{\infty} \mathscr{E}\left\{\|z(\iota)\|^{2}\right\} \leq \gamma^{2} \sum_{l=0}^{\infty} \mathscr{E}\left\{\|\omega(\iota)\|^{2}\right\}
$$

Accordingly, by means of Definition 2, one concludes that FMSSPS (16) is SS with $\mathscr{H}_{\infty}$ performance index $\gamma$. This completes the proof.

\section{Simulation Examples}

Example 1. Consider FMSSPS (16) with the following parameters:

$$
\begin{aligned}
& A_{11}=\left[\begin{array}{lll}
0.3 & 1.7 & 0.3 \\
0.8 & 0.1 & 0.3 \\
1.4 & 0.2 & 0.9
\end{array}\right], \\
& B_{11}=\left[\begin{array}{ll}
0.5 & 0.4 \\
1.2 & 0.2 \\
0.2 & 0.3
\end{array}\right], \\
& C_{11}=\left[\begin{array}{lll}
0.6 & 0.2 & 0.7
\end{array}\right]^{\top} \text {, } \\
& D_{11}=\left[\begin{array}{lll}
0.3 & 0.4 & 0.9
\end{array}\right] \text {, } \\
& M_{11}=\left[\begin{array}{ll}
0.5 & 0.8
\end{array}\right] \text {, } \\
& G_{11}=0.7 \text {, } \\
& A_{12}=\left[\begin{array}{lll}
0.1 & 0.4 & 1.1 \\
1.5 & 0.2 & 0.1 \\
0.8 & 1.1 & 0.6
\end{array}\right] \text {, } \\
& B_{12}=\left[\begin{array}{ll}
1.4 & 0.6 \\
0.7 & 0.1 \\
0.5 & 0.8
\end{array}\right], \\
& C_{12}=\left[\begin{array}{lll}
0.3 & 0.5 & 1
\end{array}\right]^{\top} \text {, } \\
& D_{12}=\left[\begin{array}{lll}
0.6 & 0.2 & 0.3
\end{array}\right] \text {, } \\
& M_{12}=\left[\begin{array}{ll}
1.5 & 0.5
\end{array}\right] \text {, } \\
& G_{12}=0.4 \text {, } \\
& A_{21}=\left[\begin{array}{lll}
0.2 & 1.1 & 0.1 \\
0.2 & 1.1 & 1.2 \\
0.6 & 0.9 & 1.1
\end{array}\right] \text {, } \\
& B_{21}=\left[\begin{array}{ll}
0.3 & 0.2 \\
0.4 & 0.5 \\
0.2 & 0.3
\end{array}\right], \\
& C_{21}=\left[\begin{array}{lll}
1.8 & 0.7 & 0.2
\end{array}\right]^{\top} \text {, } \\
& D_{21}=\left[\begin{array}{lll}
0.6 & 0.9 & 0.1
\end{array}\right] \text {, } \\
& M_{21}=\left[\begin{array}{ll}
0.3 & 0.5
\end{array}\right] \text {, } \\
& G_{21}=0.5 \text {, } \\
& A_{22}=\left[\begin{array}{lll}
1.1 & 1.5 & 2.4 \\
1.5 & 0.7 & 0.6 \\
0.1 & 1.1 & 0.8
\end{array}\right], \\
& B_{22}=\left[\begin{array}{ll}
0.6 & 0.2 \\
0.5 & 0.3 \\
0.7 & 0.5
\end{array}\right], \\
& C_{22}=\left[\begin{array}{lll}
0.6 & 0.3 & 0.4
\end{array}\right]^{\top} \text {, } \\
& D_{22}=\left[\begin{array}{lll}
0.7 & 0.6 & 0.7
\end{array}\right] \text {, } \\
& M_{22}=\left[\begin{array}{ll}
0.5 & 0.6
\end{array}\right] \text {, } \\
& G_{22}=0.3 \text {. }
\end{aligned}
$$

The TPM of the corresponding FMSSPS (1) is selected as $\Pi=\left[\begin{array}{ll}0.25 & 0.75 \\ 0.65 & 0.35\end{array}\right]$. For another MP $\Phi$ in SCP (7), the TPM is chosen as $\Phi=\left[\begin{array}{ll}0.4 & 0.6 \\ 0.5 & 0.5\end{array}\right]$.

Let $\gamma=5, \epsilon=0.002, \hbar_{1}(\xi(\iota))=\left(15-x_{2}(\iota)\right) / 30$, and $\hbar_{2}(\xi(\iota))=1-\hbar_{1}(\xi(\iota))$. In view of the LMIs of Theorem 1 , 
the controller gains $K_{q i}(q=1,2 ; f=1,2,3,4)$ can be derived as follows:

$$
\begin{aligned}
& K_{1,1}=\left[\begin{array}{lll}
-0.0008 & -0.0016 & -0.0001 \\
-0.0010 & -0.0011 & 0.0005
\end{array}\right], \\
& K_{1,2}=\left[\begin{array}{lll}
-0.0011 & -0.0063 & -0.0088 \\
-0.0010 & -0.0061 & -0.0085
\end{array}\right], \\
& K_{2,1}=\left[\begin{array}{lll}
-0.0007 & 0.0032 & 0.0022 \\
-0.0006 & 0.0017 & 0.0015
\end{array}\right], \\
& K_{2,2}=\left[\begin{array}{lll}
-0.0009 & 0.0146 & 0.0249 \\
-0.0010 & 0.0148 & 0.0244
\end{array}\right] .
\end{aligned}
$$

To carry on the simulation study, the external disturbance and the initial condition are selected as $\omega(k)=0.9$ $\exp (-0.4 \iota) \sin (50 \iota)$ and $x(0)=\left[\begin{array}{lll}-0.1 & 0.2 & 0.3\end{array}\right]^{\top}$, respectively. The possible mode switching of Markov process $\varphi(\iota)$ and the evolution of states $x_{1}(l), x_{2}(\iota)$, and $x_{3}(\iota)$ over 100 realizations are depicted in Figure 1, respectively. The control input over 100 realizations is plotted in Figure 2. Furthermore, the evolution of the output $z(l)$ over 100 realizations is shown in Figure 3.

\section{Conclusions}

In this work, the $H_{\infty}$ control problem has been discussed for FMSSPSs with the SCP. In order to coordinate the data transmission and save the bandwidth usage, the SCP with a compensator is applied to schedule the information exchange. Furthermore, some sufficient criteria have been forwarded such that the resulting system is SS. Finally, one example is exhibited to show the effectiveness and correctness of the developed results. In addition, some advanced techniques including the sliding mode-based filter will be researched in our following work [28, 29].

\section{Data Availability}

No data were utilized to support this work.

\section{Conflicts of Interest}

The authors declare that they have no conflicts of interest.

\section{Acknowledgments}

This work was supported by the Science and Technology Project of China Southern Power Grid Company Ltd. (nos. 080037KK52190037 and GZHKJXM20190108).

\section{References}

[1] L. Kang, Y. Wang, and T. Hou, "Robust control for nonlinear Markov jump systems with partially unknown transition probabilities," Mathematical Problems in Engineerin, vol. 2020, Article ID 1940676, 2020.

[2] B. Wang, J. Cheng, and X. Zhou, "A multiple hierarchical structure strategy to quantized control of Markovian switching systems," Applied Mathematics and Computation, vol. 373, Article ID 125037, 2020.
[3] Y. Wu, J. Cheng, X. Zhou, J. Cao, and M. Luo, "Asynchronous filtering for nonhomogeneous Markov jumping systems with deception attacks," Applied Mathematics and Computation, vol. 394, Article ID 125790, 2021.

[4] J. Cheng, Y. Wu, L. Xiong, J. Cao, and J. H. Park, "Resilient asynchronous state estimation of Markov switching neural networks: a hierarchical structure approach," Neural Networks, vol. 135, pp. 29-37, 2021.

[5] J. Cheng, J. H. Park, J. Cao, and W. Qi, "A hidden mode observation approach to finite-time SOFC of Markovian switching systems with quantization," Nonlinear Dynamics, vol. 100, no. 1, pp. 509-521, 2020.

[6] J. Cheng, Y. Shan, J. Cao, and J. H. Park, "Nonstationary control for T-S fuzzy Markovian switching systems with variable quantization density," IEEE Transactions on Fuzzy Systems, p. 1, 2020.

[7] J. Xiao, J. Cao, J. Cheng, S. Zhong, and S. Wen, "Novel methods to finite-times Mittag-Leffler synchronization problem of fractional-order quaternion-valued neural networks," Information Sciences, vol. 526, pp. 221-244, 2020.

[8] J. Cheng, D. Zhang, W. Qi, J. Cao, and K. Shi, "Finite-time stabilization of T-S fuzzy semi-Markov switching systems: a coupling memory sampled-data control approach," Journal of The Franklin Institute, vol. 357, no. 16, pp. 11256-11280, 2020.

[9] L. Fang, S. Ding, J. H. Park, and L. Ma, "Adaptive fuzzy control for nontriangular stochastic high-order nonlinear systems subject to asymmetric output constraints," IEEE Transactions on Cybernetics, p. 1, 2020.

[10] M.-J. Hu, J. H. Park, and Y.-W. Wang, "Stabilization of positive systems with time delay via the Takagi-Sugeno fuzzy impulsive control," IEEE Transactions on Cybernetics, p. 1, 2020.

[11] J. Cheng, W. Huang, H.-K. Lam, J. Cao, and Y. Zhang, "Fuzzymodel-based control for singularly perturbed systems with nonhomogeneous Markov switching: a dropout compensation strategy," IEEE Transactions on Fuzzy Systems, p. 1, 2020.

[12] Y. Shan, X. Liu, K. She, S. Zhong, J. Cheng, and X. Zhang, "Extended dissipative asynchronous filtering for T-S fuzzy switched systems with partial transition descriptions and incomplete measurements," Nonlinear Analysis: Hybrid Systems, vol. 37, Article ID 100906, 2020.

[13] J. Cheng, W. Huang, J. H. Park, and J. Cao, "A hierarchical structure approach to finite-time filter design for fuzzy Markov switching systems with deception attacks," IEEE Transactions on Cybernetics, p. 1, 2021.

[14] W. Assawinchaichote and S. K. Sing Kiong Nguang, "Fuzzy $\mathrm{H} / \mathrm{sub} /$ spl infin//output feedback control design for singularly perturbed systems with pole placement constraints: an LMI approach," IEEE Transactions on Fuzzy Systems, vol. 14, no. 3, pp. 361-371, 2006.

[15] J. Dong and G.-H. Yang, "control design for fuzzy discretetime singularly perturbed systems via slow state variables feedback: an LMI-based approach," Information Sciences, vol. 179, no. 17, pp. 3041-3058, 2009.

[16] G. Wang, C. Huang, Q. Zhang, and C. Yang, "Stabilisation bound of stochastic singularly perturbed systems with Markovian switching by noise control," IET Control Theory Appl, vol. 8, no. 5, pp. 367-374, 2014.

[17] H. Shen, F. Li, S. Xu, and V. Sreeram, "Slow state variables feedback stabilization for semi-markov jump systems with singular perturbations," IEEE Transactions on Automatic Control, vol. 63, no. 8, pp. 2709-2714, 2018.

[18] J. Wang, S. Ma, C. Zhang, and M. Fu, "Finite-time," IEEE Trans. Cybern.vol. 49, no. 6, pp. 2133-2143, 2019. 
[19] H. Shen, F. Li, Z. G. Wu, J. H. Park, and V. Sreeram, "Fuzzymodel based nonfragile control for nonlinear singularly perturbed systems with semi-Markov jump parameters," IEEE Trans. Fuzzy Syst.vol. 26, no. 6, pp. 3428-3439, 2018.

[20] J. Cheng, Y. Wang, J. H. Park, J. Cao, and K. Shi, "Static output feedback quantized control for fuzzy Markovian switching singularly perturbed systems with deception attacks," IEEE Transactions on Fuzzy Systems, Early Access, 2021.

[21] K. Mei, L. Ma, R. He, and S. Ding, "Finite-time controller design of multiple integrator nonlinear systems with input saturation," Applied Mathematics and Computation, vol. 372, Article ID 124986, 2020.

[22] Y. Xu, H. Su, Y. J. Pan, Z. G. Wu, and W. Xu, "Stability analysis of networked control systems with round-robin scheduling and packet dropouts," J Frankl Inst, vol. 350, no. 8, pp. 2013-2027, 2013.

[23] Y. Chen, Q. Gao, J. Cheng, K. Shi, and W. Qi, "Static output feedback control for fuzzy systems with stochastic fading channel and actuator faults," IEEE Access, vol. 8, pp. 200714-200723, 2020.

[24] G. Walsh, H. Ye, and L. Bushnell, "Stability analysis of networked control systems," IEEE Trans. Control Syst. Technol.vol. 10, no. 3, pp. 438-446, 2002.

[25] J. Song, Z. Wang, and Y. Niu, "On Ho sliding mode control under stochastic communication protocol," IEEE Transactions on Automatic Control, vol. 64, no. 5, pp. 2174-2181, 2019.

[26] Z. Cao, Y. Niu, and H. R. Karimi, "Dynamic output feedback sliding mode control for Markovian jump systems under stochastic communication protocol and its application," Int $J$ Robust Nonlinear Control, vol. 30, pp. 7307-7325, 2020.

[27] J. Cheng, J. H. Park, X. Zhao, H. R. Karimi, and J. Cao, "Quantized nonstationary filtering of network-based Markov switching RSNSs: a multiple hierarchical structure strategy," IEEE Transactions on Automatic Control, vol. 65, no. 11, pp. 4816-4823, 2020.

[28] L. Liu, W. X. Zheng, and S. Ding, "An adaptive SOSM controller design by using a sliding-mode-based filter and its application to buck converter," IEEE Transactions on Circuits and Systems-I: Regular Papers, vol. 67, no. 7, pp. 2409-2418, 2020.

[29] K. Mei and S. Ding, "Second-order sliding mode controller design subject to an upper-triangular structure," IEEE Transactions on Systems, Man and Cybernetics: Systems, vol. 51, no. 1, pp. 497-507, 2021. 